\title{
Successful multimodal therapy for an $\alpha$-fetoprotein-producing gastric cancer patient with simultaneous liver metastases
}

\author{
YU FANG, LIN WANG, NINGRONG YANG, XINLEI GONG, YU ZHANG and SHUKUI QIN \\ Department of Medical Oncology, 81st Hospital of the People's Liberation Army, Nanjing, Jiangsu 210002, P.R. China
}

Received October 12, 2014; Accepted July 28, 2015

DOI: $10.3892 / 01.2015 .3731$

\begin{abstract}
AFPGC) is considered to be a special type of stomach cancer, due its features of high malignancy, fast progression, easy transferral and a poor prognosis. No standard therapy is currently available for patients with AFPGC. In the present study, the case of a 59-year-old male diagnosed with AFPGC and simultaneous liver metastases is presented. The patient presented with abdominal bloating and multiple liver lesions were revealed upon imaging. During the course of treatment, the patient's serum AFP level increased to a maximum of 20,624.6 $\mu \mathrm{g} / 1$. The patient survived for 30 months and was ultimately treated with multimodal therapy, including surgery, chemotherapy, interventional therapy and molecular targeted therapy. Treatment with paclitaxel, irinotecan and TS-1, particularly sorafenib as a molecular targeted drug, are effective for such patients. The choice of chemotherapy regimen according to the Lauren classification and the use of oral sorafenib are likely to be novel and effective treatments for this type of stomach cancer. However, investigations should be performed to identify the gastric cancer patient population most receptive to sorafenib treatment. In addition, combined chemotherapy and molecular targeting treatment requires further study in order to determine if a synergistic effect is present. Further investigation in a large-sample study is required to confirm the validity of these results.
\end{abstract}

\section{Introduction}

Gastric cancer (GC) is the second most common cause of cancer-related mortality globally (1). $\alpha$-fetoprotein (AFP)-producing GC (AFPGC) is a special type of stomach cancer, due to its rareness, and its aggressive and malignant features. AFPGC was initially described by Bourreille et al in 1970 (2) in gastric cancer patients that exhibited elevated

Correspondence to: Professor Lin Wang, Department of Medical Oncology, 81st Hospital of the People's Liberation Army, 34 Biao Yanggongjing Street, Nanjing, Jiangsu 210002, P.R. China E-mail: wanglin81yy@163.com

Key words: gastric cancer, $\alpha$-fetoprotein, sorafenib, paclitaxel levels of serum AFP and simultaneous liver metastases. AFPGC is characterized by elevated levels of serum AFP and thus, this forms the basis for diagnosis of the disease. However, at present, the exact definition of AFPGC remains unclear (2). We hypothesize that histopathological examination is important for the diagnosis of AFPGC, as other malignancies, such as hepatitis, cirrhosis, hepatocellular carcinoma and germ cell malignancies, also produce AFP and thus, must be excluded. According to the English-language literature, AFPGC accounts for 1.3-15\% of GC cases worldwide (3-8). To the best of our knowledge, no standard therapy is currently available for patients with AFPGC and the prognosis remains extremely poor. In 1965, the Lauren classification of gastric carcinoma (9) was established, which may be applied to guide treatment choices and predict prognosis of gastric cancer patients. The present study reports the case of an AFPGC patient with simultaneous liver metastases who ultimately achieved an overall survival time of 30 months following multi-modal therapy.

\section{Case report}

A 59-year-old male with upper abdominal bloating presented to a local hospital in January 2012. The patient had no history of hepatitis and there was no significant relevant family history. Endoscopy revealed a primary lesion located in the gastric cardia. Subsequent pathological examination of biopsy specimens determined the lesion to be an adenocarcinoma. Ultrasound and computed tomography revealed multiple lesions in the liver. The liver metastases lesions were defined as unresectable lesions (Fig. 1). The patient underwent a proximal gastrectomy plus liver nodule biopsy in January 2012. Histopathological examination demonstrated moderately-differentiated adenocarcinoma (Fig. 2A), diffuse type (Lauren classification) (9). Three lymph node metastases were detected in 28 retrieved lymph nodes. Cancer tissue was found in the liver nodules. Immunohistochemical analysis revealed that epidermal growth factor receptor protein and AFP (Fig. 2B) were positively expressed. However, the tumor cells were negative for vascular endothelial growth factor (VEGF) and C-met. Fluorescence in situ hybridization revealed that HER-2 gene amplification was negative. Individual tumor target detection revealed that VEGF receptor 1 (VEGFR1) mRNA expression was low $[\geq 35.3 \%$ (low, 0.0-40.0\%; moderate, 40.1-80.0\%; high, 80.1-100.0\%)], that VEGFR2 mRNA expression was low [ $\geq 14.0 \%$ (low, $0.0-40.0 \%$; 
moderate, 40.1-80.0\%; high, 80.1-100.0\%)] and that VEGFR3 mRNA expression was moderate [ $\geq 50.3 \%$ (low, $0.0-40.0 \%$; moderate, 40.1-80.0\%; high, 80.1-100.0\%)]. The post-operative period of the patient was uneventful and the bloating in the upper abdomen disappeared.

At 1 month post-diagnosis, the patient was referred to the 81st Hospital of the People's Liberation Army (Nanjing, Jiangsu, China) for chemotherapy. The serum level of carcinoembryonic antigen (CEA) was $173.3 \mu \mathrm{g} / 1$ (normal range, 0-9.9 $\mu \mathrm{g} / \mathrm{l}$ ) and the serum AFP was not checked. Other data obtained from laboratory investigations were within the normal ranges. The patient was treated with a paclitaxel $\left(135 \mathrm{mg} / \mathrm{m}^{2}\right) /$ leucovorin calcium $\left(200 \mathrm{mg} / \mathrm{m}^{2}\right) /$ fluorouracil $\left(2.4 \mathrm{~g} / \mathrm{m}^{2}\right)$ regimen (TLF) as first-line treatment. This regimen was repeated every 3 weeks for 6 cycles. During each course of this chemotherapy, the patient suffered from grade 1 leucopenia, according to the Common Terminology Criteria for Adverse Events (version 3.0) (10). Subsequent to 6 cycles of treatment, compared with the lesions in the baseline period, the liver lesions were markedly smaller. According to the Response Evaluation Criteria in Solid Tumors criteria (version 1.1) (11), the evaluation of efficacy indicated a partial response (PR) (Fig. 3). The serum CEA level returned to normal. Thereafter, 1 cycle of Xeloda monotherapy $\left(1,250 \mathrm{mg} / \mathrm{m}^{2}\right.$ on prescription, days 1-14) was administered as maintenance treatment.

At 9 months post-diagnosis, the serum AFP level was recorded at $43.9 \mu \mathrm{g} / \mathrm{l}$ (normal range, $0-10.0 \mu \mathrm{g} / \mathrm{l}$ ). To treat the liver metastases, 4 cycles of transhepatic arterial chemotherapy and embolization (TACE)-oxaliplatin $(150 \mathrm{mg}) / \mathrm{S}-1\left(50 \mathrm{mg} / \mathrm{m}^{2}\right.$ on prescription, days 1-28) (oral) was administered. No significant adverse effects were observed and no gastrointestinal or bone marrow toxicities were detected. Following 2 cycles of treatment, the serum AFP level had decreased to normal. Computed tomography evaluation showed that the efficacy was being maintained as a PR.

At 19 months post-diagnosis, the serum level of AFP had increased to $186.8 \mu \mathrm{g} / \mathrm{l}$. Computed tomography revealed retroperitoneal lymph node swelling and new metastases to the right lung. The efficacy was now evaluated as progressive disease (PD). The patient accepted CPT-11/leucovorin calcium/fluorouracil regimen (FOLFIRI) chemotherapy. Following 2 cycles of treatment, the serum AFP level had decreased to a normal level. However, subsequent to 4 cycles of treatment, computed tomography revealed that the liver metastases had increased in size by $\sim 10 \%$ compared with previously, and the serum AFP level had increased to $340.4 \mu \mathrm{g} / 1$. The disease was considered to be undergoing slow progression and 1 cycle of an oxaliplatin/capecitabine regimen was administered as palliative chemotherapy.

At 22 months post-diagnosis, the serum AFP level had increased to $998.8 \mu \mathrm{g} / \mathrm{l}$. The patient required active treatment. By searching the literature, a single study was found on AFPGC patients who were orally administered sorafenib to extend their survival time and improve their quality of life (12). Therefore at 22 months post-diagnosis, the patient commenced treatment with oral sorafenib (400 mg/day and $200 \mathrm{mg} /$ day, alternately) monotherapy due to refusal to undertake other chemotherapies. The serum AFP level had decreased to $739.1 \mu \mathrm{g} / 12$ weeks later. At 24 months post-diagnosis, upper abdominal magnetic resonance imaging scans demonstrated that the liver lesions

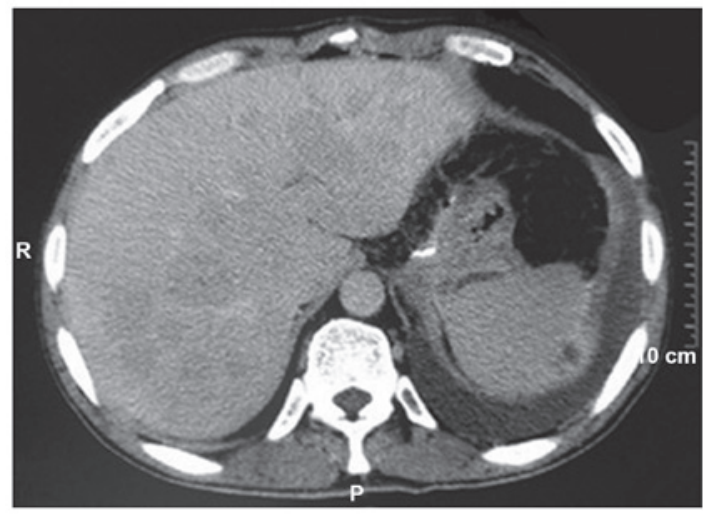

Figure 1. Abdominal contrast-enhanced computed tomography scan revealing multiple and diffuse liver metastases.

were increased in size and revealed a tumor thrombus in the left branch of the portal vein. At the same time, the serum AFP level had increased to $837.0 \mu \mathrm{g} / \mathrm{l}$. The evaluation of efficacy was PD. Sorafenib treatment was stopped. The only adverse event observed was grade 1 blood pressure.

At 27 months post-diagnosis, the patient complained of upper abdominal pain and bloating. The evaluation of efficacy was PD by upper abdominal magnetic resonance imaging scans. The serum AFP level had increased to 20,624.6 $\mu \mathrm{g} / 1$. Abraxane $\left(75 \mathrm{mg} / \mathrm{m}^{2}\right.$ on days 1 and 8$)$ monotherapy was administered. Following the first cycle of treatment, the serum AFP level had decreased to $9,392.2 \mu \mathrm{g} / \mathrm{l}$. The symptoms of upper abdominal pain and bloating were also alleviated. Thereafter, a second cycle of treatment was performed.

At 29 months post-diagnosis, the patient presented with jaundice and a fever. Laboratory examination results were as follows: Total bilirubin, $132.1 \mu \mathrm{mol} / \mathrm{l}$ (normal range, 5.1-19.0 $\mu \mathrm{mol} / \mathrm{l}$ ); direct bilirubin, $114.8 \mu \mathrm{mol} / 1$ (normal range, 1.7-6.8 $\mu \mathrm{mol} / \mathrm{l}$ ); albumin, $29.3 \mathrm{~g} / 1$ (normal range, 40.0-55.0 g/l); aspartate aminotransferase, $139.0 \mathrm{IU} / 1$ (normal range, 0-39.0 IU/1); and urobilirubin and urobilinogen were positive. Abdominal ultrasound revealed biliary sludge. A diagnosis of hepatocellular jaundice with cholestatic jaundice was formed. Unexpectedly, the serum AFP level had decreased to 4,225.73 $\mu \mathrm{g} / \mathrm{l}$. Magnetic resonance cholangiopancreatography demonstrated that compared with previously, the hepatic nodular lesions were smaller, the right hepatic duct was dilated, and the right lung nodules were smaller (Fig. 4). Following symptomatic and supportive treatment, such as magnesium isoglycyrrhizinate, reduced glutathione and Kuhuang injection, the bilirubin progressively increased and the indication for chemotherapy was lost. Multidisciplinary consultation recommended that the patient should undergo endoscopic retrograde cholangiopancreatography plus stent implantation. However, the patient refused and went home to recuperate. The patient succumbed to the disease at 30 months post-diagnosis.

\section{Discussion}

AFPGC is a special type of GC, accounting for only 1.3-15\% of GCs in the English-language literature (3). AFPGC was first described in 1970 by Bourreille et al (2). Elevated serum AFP level is the basis for the diagnosis of AFPGC, while it is also 


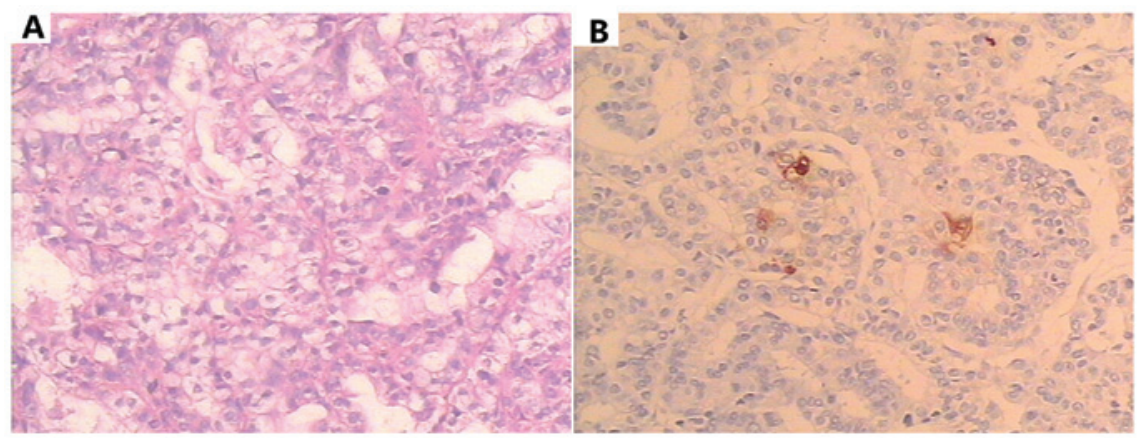

Figure 2. Histology of the primary tumour: (A) Histopathological examination revealing moderately-differentiated adenocarcinoma components (hematoxylin and eosin staining; magnification, x100). (B) Immunohistological staining showing positive result for $\alpha$-fetoprotein in the gastric tumor (Envision; magnification, $\mathrm{x} 100)$.
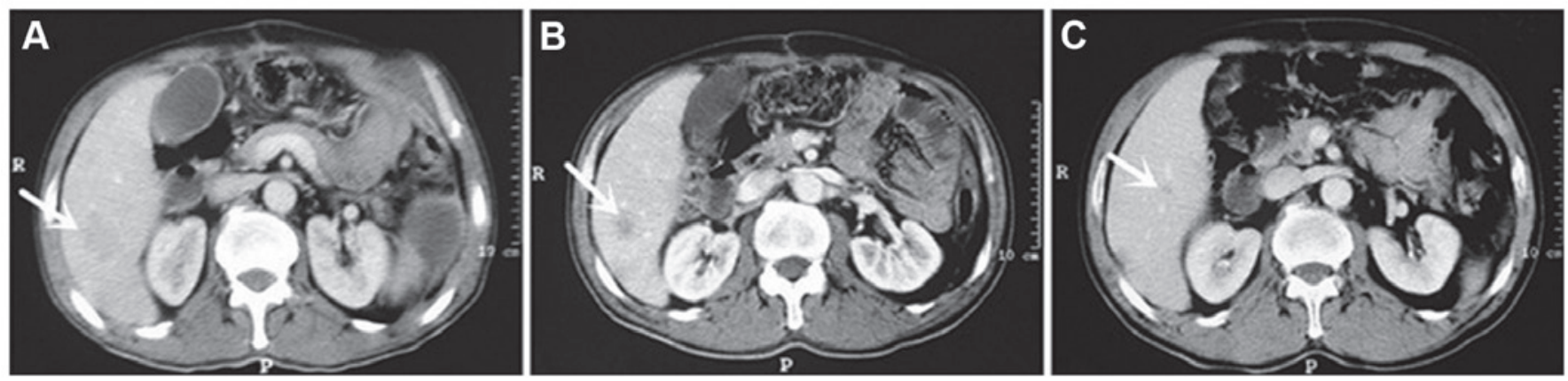

Figure 3. Abdominal contrast-enhanced computed tomography scans of the patient (A) prior to, and at (B) 2 and (C) 3 months after the initiation of treatment with paclitaxel/leucovorin calcium/fluorouracil regimen chemotherapy, showing the shrinking liver metastases.
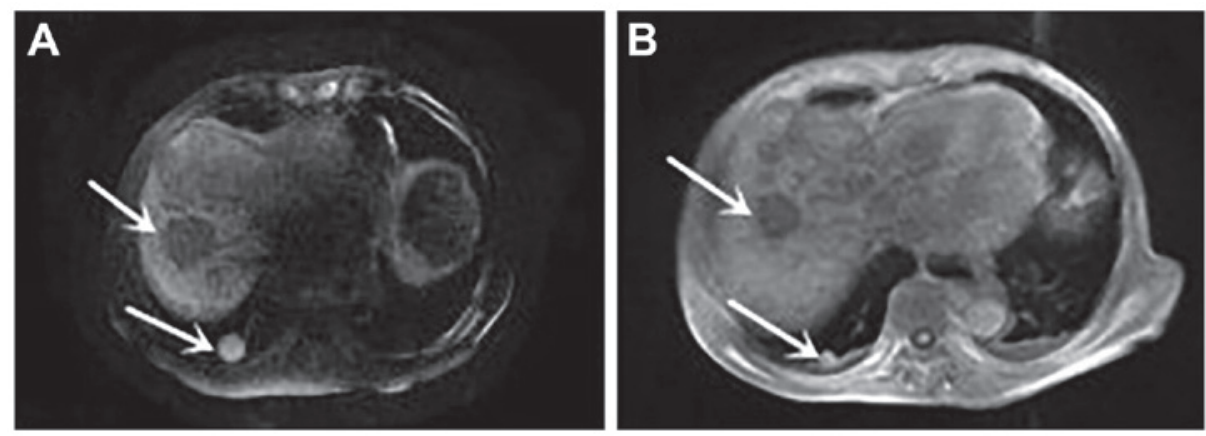

Figure 4. Upper abdominal magnetic resonance imaging of the patient (A) prior to and at (B) 2 months after initiation of treatment with Abraxane, showing the shrinking liver and lung metastases.

required to rule out other possible diseases, such as hepatitis, cirrhosis, hepatocellular carcinoma and germ cell malignancy. This type of GC is prone to liver and lymph node metastasis, and has a poor prognosis. There is no effective means to treat AFPGC, particularly in the field of internal medicine. Currently, the disease is treated with reference to common cancer, but the prognosis remains extremely poor. The median survival time of AFPGC patients is significantly shorter than for those with normal GC (13).

The present study reports the case of an AFP-positive GC patient with simultaneous liver metastases. The detection of serum AFP level was not considered at first. This also suggests that attention should be focused on the disease in the future process of diagnosis and treatment. The detection of serum

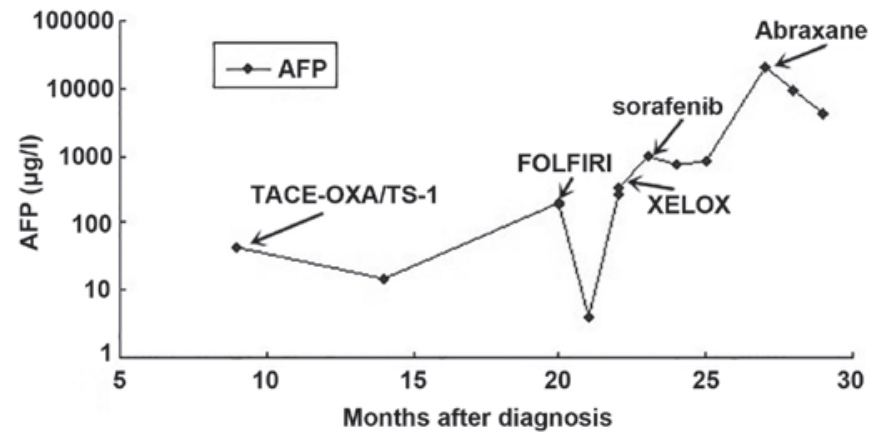

Figure 5. Therapeutic course. AFP, $\alpha$-fetoprotein; TACE-OXA, transhepatic arterial chemotherapy and embolization; OXA, oxaliplatin; TS-1, tegafur/gimeracil/oteracil potassium; FOLFIRI, CPT-11/leucovorin calcium/fluorouracil; XELOX, oxaliplatin/capecitabine. 
AFP level should be applied as a routine examination in GC patients, particularly those with liver metastases. In the post-treatment process of the present study, the serum AFP level was constantly monitored, and the rise and fall in serum AFP levels were found to be positively correlated with the patient's condition. This is therefore an important means that can be used to evaluate condition changes of a patient.

According to studies, the different Lauren classifications of GC have different sensitivity to chemotherapeutic drugs. The patients who are of the diffuse type according to the Lauren classification can benefit more from drugs such as paclitaxel, irinotecan and S-1 (14-17). Fukuda et al reported that of the eight AFPGC patients that received chemotherapy, two patients who received cisplatin plus paclitaxel therapy exhibited a PR (18). Of the six remaining patients, who received oral TS-1, in combination with either cisplatin or camptothecin, the best response achieved was stable disease (18). In the present case, the patient' disease was of diffuse type. First-line chemotherapy with a paclitaxel-based TLF regimen was selected and the efficacy achieved was a PR. Following the use of this type of drug for 2 years, Abraxane (paclitaxel) was used, which again shrank the tumor and decreased the serum AFP level. The drug exhibited good antitumor effect. This encourages us not to give up when treating AFPGC patients. In addition, the application of irinotecan for AFPGC patients has also been reported (19). In this case, the patients were treated with irinotecan-based FOLFIRI regimen, which was also effective. Paclitaxel-based chemotherapy or irinotecan-based chemotherapy are valid for use in such patients. This situation, whether associated with the Lauren classification or affected by other factors, requires further study.

The liver metastatic lesions were defined as unresectable lesions in the present case. TACE-oxaliplatin/S-1 (oral) therapy was performed and achieved tumor shrinkage and AFP level reduction. This shows the value of interventional therapy. A survival time of $>5$ years has also been reported in an AFPGC patient receiving hepatic arterial infusion therapy (12). It was suggested that hepatic arterial infusion therapy may improve the prognosis compared with the use of systemic chemotherapies in AFPGC patients with multiple liver metastases (12).

Sorafenib is a multi-kinase inhibitor, but its specific targets are not fully understood. On the one hand, it can inhibit the activity of the hepatocyte growth factor/c-Met pathway and the downstream RAF/mitogen-activated protein kinase kinase/extracellular signal-regulated kinases signaling pathway, resulting in inhibition of tumor growth. On the other hand, it can block tumor angiogenesis by inhibiting VEGFR and platelet-derived growth factor receptor to indirectly inhibit the growth of tumor cells. The drug has achieved impressive efficacy in hepatocellular carcinoma $(20,21)$. During an Oriental Studies subgroup analysis, in which AFP-positive HCC patients (serum AFP level, $\geq 40 \mu \mathrm{g} / \mathrm{l}$ ) were treated with sorafenib and placebo, respectively, the median survival time was prolonged by 1.8 months in the sorafenib group compared with the placebo group (5.9 vs. 4.1 months; hazard ratio, 0.65) (22). AFPGC and AFP-positive HCC are similar with regard to the elevated serum AFP level. Studies have reported the use of sorafenib for the treatment of advanced GC (23), and even AFPGC (12), revealing a certain extension in the patient's survival time and improvement to their quality of life. In the present case, immunohistochemistry revealed that c-Met and VEGF were negative, and that the VEGFRs were mainly expressed at a low level. This may be one of the reasons why sorafenib monotherapy only reduced the level of AFP and a decrease in tumor size was not observed. Therefore, we believe that sorafenib in AFPGC may be effective. Thus, investigations should be performed to identify the gastric cancer patient population most receptive to sorafenib treatment. In addition, the effect may be more significant if combining other chemotherapies with sorafenib.

In summary, AFPGC is a rare, aggressive and malignant tumor. In the present case, it was treated with multimodal therapy, including surgery, chemotherapy, interventional therapy and molecular targeted therapy. The use of a paclitaxel-based TLF regimen, a irinotecan-based FOLFIRI regimen, sorafenib and intervention therapy was valid. The study showed that antitumor therapy is active and effective. The choice of chemotherapy regimen according to the Lauren classification and the use of oral sorafenib are likely to be novel and effective treatments for this type of stomach cancer. However, investigations should be performed to identify the gastric cancer patient population most receptive to sorafenib treatment. In addition, combined chemotherapy and molecular targeting treatment require further study to determine if there is a synergistic effect. This case study may indicate a potential treatment option for this rare disease. Consideration of this type of cancer should be ensured in future clinical practice.

\section{Acknowledgements}

The authors are grateful to the patient and his family for their permission to report the present case study. This study was financed by a Grant-in-aid for Gastric Cancer Research from the Chinese Gastrointestinal Oncology Group (no. 20130101002).

\section{References}

1. Parkin DM, Bray F, Ferlay J and Pisani P: Global cancer statistics, 2002. CA Cancer J Clin 55: 74-108, 2005.

2. Bourreille J, Metayer P, Sauger F, Matray F and Fondimare A: Existence of alpha fetoprotein during gastric-origin secondary cancer of the liver. Presse Med 78: 1277-1278, 1970 (In French).

3. Hirajima S, Komatsu S, Ichikawa D, Kubota T, Okamoto K, Shiozaki A, Fujiwara H, Konishi H, Ikoma H and Otsuji E: Liver metastasis is the only independent prognostic factor in AFP-producing gastric cancer. World J Gastroenterol 19: 6055-6061, 2013.

4. Kono K, Amemiya H, Sekikawa T, Iizuka H, Takahashi A Fujii $\mathrm{H}$ and Matsumoto Y: Clinicopathologic features of gastric cancers producing alpha-fetoprotein. Dig Surg 19: 359-365, 2002.

5. Chang YC, Nagasue N, Kohno H, Taniura H, Uchida M, Yamanoi A, Kimoto T and Nakamura T: Clinicopathologic features and long-term results of alpha-fetoprotein-producing gastric cancer. Am J Gastroenterol 85: 1480-1485, 1990.

6. Chun $\mathrm{H}$ and Kwon SJ: Clinicopathological characteristics of alpha-fetoprotein-producing gastric cancer. J Gastric Cancer 11: 23-30, 2011.

7. Liu X, Cheng Y, Sheng W, Lu H, Xu Y, Long Z, Zhu H and Wang Y: Clinicopathologic features and prognostic factors in alpha-fetoprotein-producing gastric cancers: Analysis of 104 cases. J Surg Oncol 102: 249-255, 2010.

8. McIntire KR, Waldmann TA, Moertel CG and Go VL: Serum alpha-fetoprotein in patients with neoplasms of the gastrointestinal tract. Cancer Res 35: 991-996, 1975. 
9. Lauren P: The two histological main types of gastric carcinoma: diffuse and so-called intestinal-type carcinoma. An attempt at a histo-clinical classification. Acta Pathol Microbiol Scand 64: 31-49, 1965.

10. National Cancer Institute: Common Terminology Criteria for Adverse Events v3.0 (CTCAE). http://ctep.cancer.gov/protocolDevelopment/electronic_applications/docs/ctcaev3.pdf. Accessed June 16, 2014.

11. Therasse P, Arbuck SG, Eisenhauer EA, Wanders J, Kaplan RS, Rubinstein L, Verweij J, Van Glabbeke M, van Oosterom AT, Christian MC and Gwyther SG: New guidelines to evaluate the response to treatment in solid tumors. European Organization for Research and Treatment of Cancer, National Cancer Institute of the United States, National Cancer Institute of Canada. J Natl Cancer Inst 92: 205-216, 2000.

12. Koneri K, Hirono Y, Fujimoto D, Sawai K, Morikawa M, Murakami M, Goi T, Iida A, Katayama K and Yamaguchi A: Five-year survival of alpha-fetoprotein producing gastric cancer with synchronous liver metastasis: A case report. J Gastric Cancer 13: 58-64, 2013.

13. Lew DH, Jung WT, Kim HJ, Min HJ, Ha CY, Kim HJ, Kim TH and Ko GH: Clinicopathological characteristics and prognosis of alpha-fetoprotein producing gastric cancer. Korean J Gastroenterol 62: 327-335, 2013 (In Korean).

14. Yamaguchi K, Tada M, Horikoshi N, Otani T, Takiuchi $H$ Saitoh S, Kanamaru R, Kasai Y, Koizumi W, Sakata Y, et al: Phase II study of paclitaxel with 3-h infusion in patients with advanced gastric cancer. Gastric Cancer 5: 90-95, 2002.

15. Emi Y, Yamamoto M, Takahashi I, Orita H, Kakeji Y, Kohnoe S and Maehara Y: Phase II study of weekly paclitaxel by one-hour infusion for advanced gastric cancer. Surg Today 38: 1013-1020, 2008.

16. Narahara $H$, Iishi $H$, Imamura $H$, Tsuburaya $A$, Chin $K$, Imamoto $H$, Esaki T, Furukawa H, Hamada C and Sakata Y: Randomized phase III study comparing the efficacy and safety of irinotecan plus S-1 with S-1 alone as first-line treatment for advanced gastric cancer (study GC0301/TOP-002). Gastric Cancer 14: 72-80, 2011.
17. Ajani JA, Rodriguez W, Bodoky G, Moiseyenko V, Lichinitser M, Gorbunova V, Vynnychenko I, Garin A, Lang I and Falcon S: Multicenter phase III comparison of cisplatin/S-1 with cisplatin/infusional fluorouracil in advanced gastric or gastroesophageal adenocarcinoma study: The FLAGS trial. J Clin Oncol 28: 1547-1553, 2010.

18. Fukuda K, Ito S, Shimizu K, Mikami E, Sakuraba S, Shiroki T, Ikehata A, Murakami A, Ono S, Sakuma T and Kato S: Retrospective analysis concerning AFP-producing gastric cancer. Gan To Kagaku Ryoho 40: 191-195, 2013 (In Japanese).

19. Shiochi H, Yamada M, Kishina M, Murawaki Y, Miura M, Azumi T, Yuuki T, Tanaka S, Kono M, Yoshimura T, et al: A case of AFP-producing gastric cancer responding to the combination of systemic chemotherapy, transcatheter arterial embolization and hepatic infusion chemotherapy. Gan To Kagaku Ryoho 36: 843-846, 2009 (In Japanese).

20. Llovet JM, Ricci S, Mazzaferro V, Hilgard P, Gane E, Blanc JF, de Oliveira AC, Santoro A, Raoul JL, Forner A, et al: Sorafenib in advanced hepatocellular carcinoma. N Engl J Med 359: 378-390, 2008.

21. Cheng AL, Kang YK, Chen Z, Tsao CJ, Qin S, Kim JS, Luo R, Feng J, Ye S, Yang TS, et al: Efficacy and safety of sorafenib in patients in the Asia-Pacific region with advanced hepatocellular carcinoma: A phase III randomised, double-blind, placebo-controlled trial. Lancet Oncol 10: 25-34, 2009.

22. Cheng AL, Guan Z, Chen Z, Tsao CJ, Qin S, Kim JS, Yang TS, Tak WY, Pan H, Yu S, et al: Efficacy and safety of sorafenib in patients with advanced hepatocellular carcinoma according to baseline status: Subset analyses of the phase III Sorafenib Asia-Pacific trial. Eur J Cancer 48: 1452-1465, 2012.

23. Kim C, Lee JL, Choi YH, Kang BW, Ryu MH, Chang HM, Kim TW and Kang YK: Phase I dose-finding study of sorafenib in combination with capecitabine and cisplatin as a first-line treatment in patients with advanced gastric cancer. Invest New Drugs 30: 306-315, 2012. 